\section{SAT0107 SEQUENTIAL ULTRASOUND SHOWS A LATE INCREASE IN INFLAMMATORY BURDEN IN ANTI-CCP POSITIVE PATIENTS WITH NON-SPECIFIC MUSCULOSKELETAL SYMPTOMS JUST BEFORE PROGRESSION TO INFLAMMATORY ARTHRITIS.}

P. Pentony ${ }^{1,2, *}$, K. Mankia ${ }^{1,2}$, E. M. Hensor ${ }^{1}$, J. L. Nam ${ }^{1,2}$, L. Hunt ${ }^{1,2}$, L. GarciaMontoya $^{2,3}$, L. Duquenne ${ }^{2,3}$, P. Emery ${ }^{1,2} .{ }^{1}$ Leeds Institute of Rheumatic and Musculoskeletal Medicine, University of Leeds; ${ }^{2}$ Leeds Biomedical Research Centre, National Institute of Health Research; ${ }^{3}$ Leeds Institute of Rheumatic and Musculoskeletal Medicine, Leeds University, Leeds, United Kingdom

Background: US abnormalities occur in patients with new rheumatoid arthritis (RA) and can also predict the development of clinical synovitis in anti-CCP positive patients with musculoskeletal symptoms (MSK) (CCP+) [1]. However, the timing of development of US synovitis in relation to clinical synovitis has not been longitudinally studied.

Objectives: To investigate whether US synovitis (grey scale (GS) and power doppler (PD)) changes in the period prior to the development of inflammatory arthritis (IA) in CCP+ individuals compared to those who do not develop IA.

Methods: $\mathrm{CCP}+$ individuals were prospectively followed until the development of clinical synovitis in at least one joint (progressors). Progressors were compared to $\mathrm{CCP}+$ individuals who did not develop clinical synovitis (non-progressors). For all subjects, US scans were performed at baseline, 6 and 12 months and then annually, and/or at the time of onset of clinical synovitis. A 22 paired joint US score was calculated for PD and GS >1 using the wrists, MCPJs (1-5) and PIPJs (1-5). Comparisons were made between baseline, the ultrasound prior to progression (or time equivalent in non-progressors)(scan 2) and progression scan (or time-equivalent) (scan 3).

Results: Patients with at least 3 serial US were included: $22 \mathrm{CCP}+$ progressors and $22 \mathrm{CCP}+$ non progressors. Age and gender was similar between groups.

The majority of patients in both groups showed no change in PD or GS $>1$ between baseline and scan 2 (table 1). All 22 non-progressors (100\%) had a PD score of 0 at baseline, compared with 16/22 (72.7\%) progressors. In contrast, between scan 2 and 3 (progression scan), the majority of patients in the progressor group increased total PD and GS scores, while non-progressors remained the same (table 1). Time between scan 2 and 3 was similar between groups.

Table 1. Direction of change in 22 paired joint PD and GS>1 scores between baseline and scan 1 and between interim scan and scan 3 .

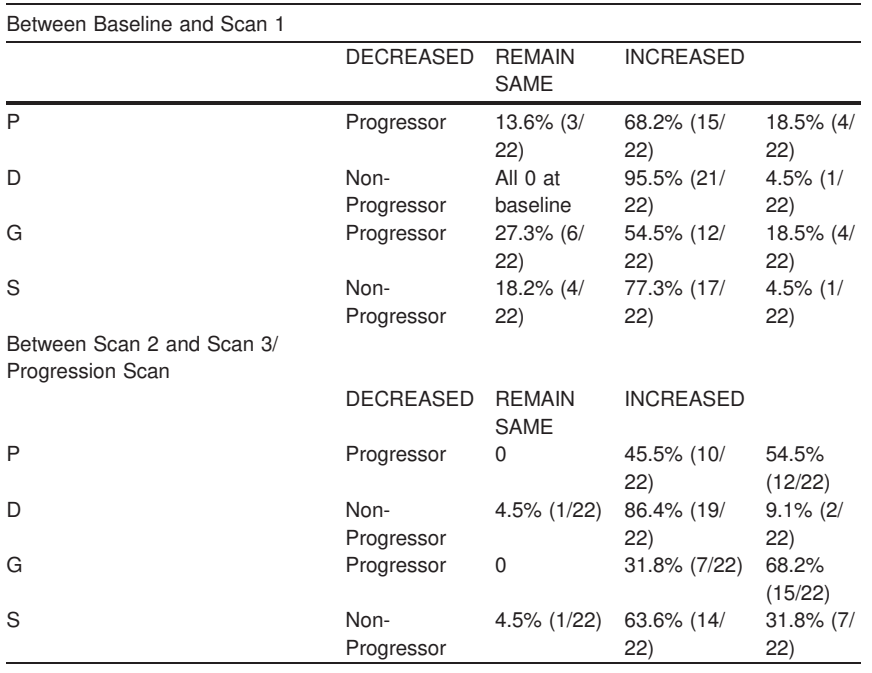

Conclusions: After a period of stability the burden of US inflammation in CCP+ patients that progress to IA increases directly before the development of IA. This later increase in US inflammation may reflect a second hit in these at risk patients occurring prior to progression to IA. This may suggest that an intervention aiming to prevent the development of IA should target the subclinical phase prior to an escalation in US inflammation.

\section{REFERENCE:}

[1] Nam JL, et al. Ultrasound findings predict progression to inflammatory arthritis in anti-CCP antibody-positive patients without clinical synovitis. Ann Rheum Dis 2016;75(12):2060-2067.

Disclosure of Interest: None declared DOI: 10.1136/annrheumdis-2018-eular.6892

\section{SAT0108 \\ EFFICACY OF ABATACEPT VERSUS ADALIMUMAB ON THE PROPORTION OF PATIENTS WITH SEROPOSITIVE, EROSIVE EARLY RA ACHIEVING DAS28 (CRP) $<2.6$ OR VALIDATED MEASURES OF REMISSION: A POST HOC ANALYSIS OF THE 2-YEAR AMPLE TRIAL}

R. Fleischmann ${ }^{1}$, M. E. Weinblatt ${ }^{2}$, H. A. Ahmad ${ }^{3}$, M. A. Maldonado ${ }^{3}$, E. Alemao ${ }^{3}$, Y. Elbez ${ }^{4}$, M. Schiff ${ }^{5} .{ }^{1}$ University of Texas Southwestern Medical Center, Dallas; ${ }^{2}$ Brigham and Women's Hospital, Boston; ${ }^{3}$ Bristol-Myers Squibb, Princeton, United States; ${ }^{4}$ Excelya, Boulogne-Billancourt, France; ${ }^{5}$ University of Colorado, Denver, United States

Background: Binary cut-offs for disease activity are frequently used in clinical decision-making for patients with RA because they are accurate reflections of disease activity, discriminate well between disease acvitiy states and are feasible to perform in routine clinical practice. ${ }^{1}$ Results from a previous post hoc analysis of the randomized, controlled, head-to-head AMPLE trial (NCT00929864) indicated a trend for increased efficacy, as assessed using DAS28 (CRP), for abatacept (ABA) compared with the TNF inhibitor adalimumab (ADA) in patients with seropositive, erosive early RA. ${ }^{2,3}$

Objectives: To evaluate post hoc the impact of treatment with either SC ABA or ADA on sustained validated definitions of remission and DAS28 (CRP) $<2.6$ in patients with seropositive, early, rapidly progressing RA with inadequate response to MTX

Methods: This post hoc analysis of the AMPLE trial builds on previous work ${ }^{3}$ to compare clinical outcomes between treatment groups in two subsets: patients with disease duration $\leq 6$ months, RF or anti-citrullinated protein antibody seropositivity and $>1$ radiographic erosion (Cohort 1 ), and patients in whom $\geq 1$ of these inclusion criteria were absent (Cohort 2). Disease activity and patient-reported outcomes were evaluated at Weeks 26, 52 and 104. Endpoints were defined as percentages of patients with DAS28 (CRP) $<2.6$, SDAl $\leq 3.3, \mathrm{CDAl}<2.8$ or Boolean remission. Endpoints were compared between $A B A$ and ADA treatment groups using chi-square tests.

Results: Of 646 randomized patients, 83 were included in Cohort 1 (ABA, $n=38$ $A D A, n=45$ ) and 563 in Cohort 2 ( $A B A, n=280 ; A D A, n=283$ ). At Week 52, significantly more ABA- than ADA-treated patients achieved DAS28 (CRP) $<2.6$ in Cohort $1(p=0.03)$, a trend that was not seen at Week 104 or in Cohort 2 at either time point. At Weeks 52 and 104, more ABA- than ADA-treated patients achieved CDAl, SDAl and Boolean remission in Cohort 1, a trend not seen in Cohort 2.

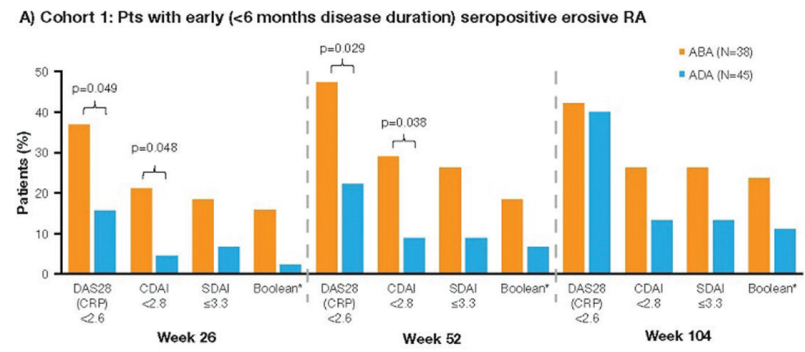

B) Cohort 2: Pts with the absence of any 1 factor in Cohort 1

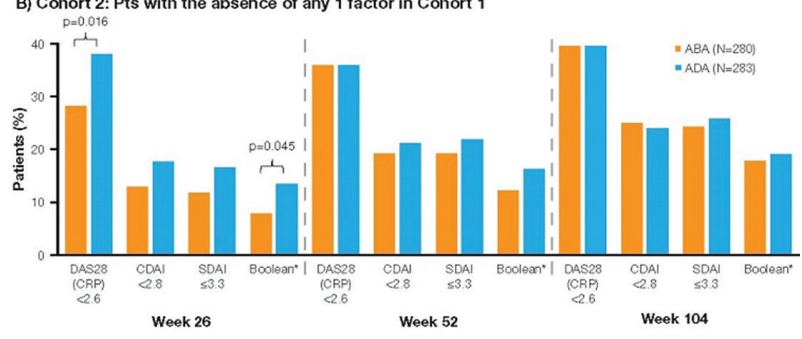

All $p>0.05$ unless otherwise stated. "Boolean remission is defined as tender joint count $\leq 1$, sollen joint count $\leq 1, \mathrm{CRP} \leq 1 \mathrm{mg} / \mathrm{dL}$ and patient global assessment $\leq 1$ (on a $0-10$ scale).

Figure 1. Comparison of Treatments by Sustained Remission Outcomes and DAS28 (CRP) $<2.6$ A) Cohort 1: Pts with early (<6 months disease duration) seropositive erosive RA. B) Cohort 2: Pts with the absence of any 1 factor in Cohort 1. All $p>0.05$ unless otherwise stated. *Boolean remission is defined as tender joint count $\leq 1$, swollen joint count $\leq 1$, $\mathrm{CRP} \leq 1 \mathrm{mg} / \mathrm{dL}$ and patient global assessment $\leq 1$ (on a $0-10$ scale).

Conclusions: This post hoc analysis indicates a trend towards increased efficacy for abatacept compared with adalimumab on measures of sustained remission and DAS28 (CRP) <2.6 in patients with seropositive, erosive early RA. These results, along with results from other studies, ${ }^{4,5}$ support the pursuit of a clinically definable subset of patients who may respond differentially to targeted therapies. 


\section{REFERENCES:}

[1] Anderson J, et al. Arthritis Care Res (Hoboken) 2012;64:640-7.

[2] Schiff M, et al. Ann Rheum Dis 2014;73:86-94

[3] Fleischmann R, et al. EULAR 2017; poster SAT0041.

[4] Harrold LR, et al. J Rheumatol 2018;45:32-9.

[5] Emery P, et al. Ann Rheum Dis 2015;74:19-26.

Disclosure of Interest: R. FleischmannGrant/research support from: AbbVie, Amgen, AstraZeneca, Bristol-Myers Squibb, Celgene, EMD-Serono, Eli Lilly, Merck, Novartis, Pfizer, Roche, Sanofi-Genzyme, UCB, Consultant for: AbbVie, ACEA, Amgen, Bristol-Myers Squibb, GSK, Eli Lilly, Novartis, Pfizer, Sanofi-Genzyme, UCB, M. Weinblatt Grant/research support from: Bristol-Myers Squibb, Amgen, Crescendo Bioscience, Sanofi, Consultant for: Bristol-Myers Squibb, Amgen, Crescendo Bioscience, AbbVie, Eli Lilly, Pfizer, Roche, Merck, Samsung, Novartis, H. Ahmad Shareholder of: Bristol-Myers Squibb, Employee of: BristolMyers Squibb, M. Maldonado Shareholder of: Bristol-Myers Squibb, Employee of: Bristol-Myers Squibb, E. Alemao Shareholder of: Bristol-Myers Squibb, Employee of: Bristol-Myers Squibb, Y. Elbez Consultant for: Bristol-Myers Squibb, M. Schiff Consultant for: Abbvie, Bristol-Myers Squibb, Eli Lilly, Johnson \& Johnson, UCB, Speakers bureau: AbbVie, Bristol-Myers Squibb DOI: 10.1136/annrheumdis-2018-eular.2333

\section{SAT0109 ULTRASONIC EVALUATION OF JOINT INVOLVEMENT IN ESTABLISHED RA: ACTIVE SYNOVITIS PREDICTS SUSTAINED TREATMENT CHANGES IN SUSPECTED BIOLOGIC FAILURE}

R.L. Batten ${ }^{1,2}$, O. Mohammed ${ }^{2}$, L. Probert ${ }^{2}$, D. Wright ${ }^{2}$, I. Atchia ${ }^{3} .{ }^{1}$ Rheumatology, Newcastle upon Tyne Hospitals NHS Foundation Trust, Newcastle;

${ }^{2}$ Rheumatology, City Hospitals Sunderland, Sunderland; ${ }^{3}$ Rheumatology, Northumbria Healthcare NHS Foundation Trust, Newcastle, United Kingdom

Background: EULAR guidelines recommend treatment of rheumatoid arthritis (RA) targeted to remission. ${ }^{1}$ Biologic switching should be considered where there is at least moderate disease activity (DAS28 >3.2). The role of ultrasound (US) in guiding treatment is perhaps less clear. D'Agostino et al have proposed a novel algorithm based on current best evidence. ${ }^{2}$

Objectives: We present a case series of 30 RA patients with perceived biologic failure. By applying the algorithm to this group of patients we reviewed the impact of musculoskeletal ultrasound findings on treatment changes when compared to DAS28 assessment alone.

Methods: All patients had US of the Backhaus 7 joints on the most affected side, and any additional symptomatic joints. A global OMERACT-EULAR synovitis score (GLOESS) was calculated for each patient. ${ }^{3}$ DAS28 was calculated at the time of US, and clinician opinion to continue or switch biologic was documented pre and post US. Patient notes were reviewed at 6 months to assess whether treatment changes were sustained.

Results: 26 patients had DAS28 $>3.2$. Of these, 10 were found to have GLOESS $>6$ and subsequently switched biologic therapy. 4 patients had DAS $28<3.2$, despite clinical suspicion of persistent inflammatory disease. Of these patients, 2 were found to have GLOESS $>6$, and subsequently switched to an alternative biologic. At 6 months 20/24 patient's management remained consistent with previous US findings, 2 patients escalated treatment despite a previously negative US, 1 patient declined escalation (although US showed synovitis), 1 patient switched due to intolerance and 6 were lost to follow-up.

Table 1. Proposed treatment outcome (switch biologics) in symptomatic patients based on clinician opinion, DAS28 and US findings

\begin{tabular}{|c|c|c|c|}
\hline & $\begin{array}{l}\text { Clinician opinion pre- } \\
\text { US }\end{array}$ & $\begin{array}{l}\text { DAS28 at time of } \\
\text { US }\end{array}$ & $\begin{array}{l}\text { Clinician opinion post- } \\
\text { US }\end{array}$ \\
\hline Switch Biologics & 23 & 26 (DAS28 >3.2) & $12(\text { GLOESS }>6)^{\mathrm{a}}$ \\
\hline $\begin{array}{l}\text { Don't Switch } \\
\text { Biologics }\end{array}$ & 7 & 4 (DAS28 <3.2) & $18($ GLOESS<6) \\
\hline $\begin{array}{l}\text { Total } \\
\text { P value }\end{array}$ & 30 & 30 & $\begin{array}{l}30 \\
<0.01^{\mathrm{b}},<0.01^{\mathrm{c}}\end{array}$ \\
\hline
\end{tabular}

a 1 patient GLOESS $<6$ with severe tenosynovitis ${ }^{\mathrm{b}}$ Fisher's Exact test ${ }^{\mathrm{C}} \mathrm{Chi}$ squared

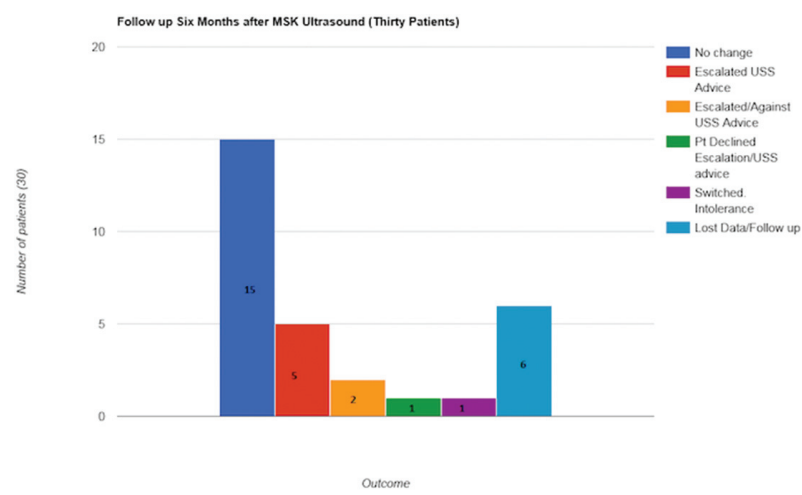

Conclusions: US significantly reduced the need to switch treatment in this cohort of patients compared with DAS28. Longitudinal follow up supports the validity of US to determine those with active disease whilst on a biologic. The use of US may prevent over-treatment, and subsequently reduce morbidity and financial cost. Further work is needed to evaluate the clinical impact and cost effectiveness of routine US prior considering a change in biologic therapy.

\section{REFERENCES:}

[1] Smolen JS, et al. Treating rheumatoid arthritis to target: recommendations of an international task force. Ann Rheum Dis 2010;69:631-7.

[2] D'Agostino MA, et al. Novel algorithms for the pragmatic use of ultrasound in the management of patients with rheumatoid arthritis: from diagnosis to remission. Annals of the Rheumatic Diseases 2016;75:1902-1908.

[3] D'Agostino MA. Exploring a new ultrasound score as a clinical predictive tool in patients with rheumatoid arthritis starting abatacept: results from the APPRAISE study. RMD Open 2016 May;5;2(1).

Disclosure of Interest: None declared

DOI: 10.1136/annrheumdis-2018-eular.4264

\section{SAT0110 TRAJECTORIES OF FUNCTIONAL DISABILITY IN PATIENTS WITH EARLY INFLAMMATORY POLYARTHRITIS AND MODERATE DISEASE ACTIVITY: RESULTS FROM THE EARLY RHEUMATOID ARTHRITIS NETWORK AND NORFOLK ARTHRITIS REGISTER}

S. Norton ${ }^{1,2}$, J. Gwinnutt ${ }^{3}$, Y. Pan ${ }^{3}$, D. Symmons ${ }^{3}$, A. MacGregor ${ }^{4}$, A. Young ${ }^{5}$, P. Kiely ${ }^{6}$, D. Walsh ${ }^{7}$, S. Verstappen ${ }^{3} .{ }^{1}$ Psychology Department, ${ }^{2}$ Academic Rheumatology, King's College London, London; ${ }^{3}$ Arthritis Research UK Centre for Epidemiology, University of Manchester, Manchester, ${ }^{4}$ Norwich Medical School, University of East Anglia, Norwich; ${ }^{5}$ School of Life and Medical Sciences, University of Hertfordshire, Hatfield; ${ }^{6}$ Rheumatology, St Georges University Hospitals NHS Foundation Trust, London; ${ }^{7}$ Arthritis Research UK Pain Centre, University of Nottingham, Nottingham, United Kingdom

Background: A large group of patients with inflammatory polyarthritis (IP), and its subset rheumatoid arthritis (RA), have moderate disease activity, despite disease modifying therapy. Identifying patients with moderate disease who are likely to have subsequent high disability may prompt different treatment strategies for these patients.

Objectives: To identify common trajectories of disability progression in patients with moderate disease in two large prospective observational studies.

Methods: The Early Rheumatoid Arthritis Network (ERAN) recruited 1236 patients with RA ( $<36$ months symptoms) from 23 centres in England from 2002 11. The Norfolk Arthritis Register (NOAR) recruited 1054 IP patients ( $<24$ months symptoms) from Norfolk, England, from 2000-8. At baseline and subsequent follow-ups, functional disability was assessed using the Health Assessment Questionnaire (HAQ). Included patients scored $\geq 3.2$ and $<5.1$ on the Disease Activity Score (DAS28) at either baseline, year 1 or year 2 , and had previously received csDMARDS ( $\mathrm{N}_{\mathrm{ERAN}}=605 ; \mathrm{N}_{\mathrm{NOAR}}=407$ ). Latent class growth models (LCGMs) were used to identify $\mathrm{HAQ}$ trajectories independently in each cohort. Age, sex, fulfilment of ACR RA criteria, symptom duration, DMARDs at baseline, and baseline 